\title{
A Case of Penetrating Aortic Atherosclerotic Ulcer with Hemoptysis
}

\author{
Masato FuKUSHIMA, MD, Yoshihiko SEINO, MD, Masatomo YoshIKAwA, MD, \\ Youichi UEDA, MD, and Teruo TAKANO, MD
}

\begin{abstract}
SUMMARY
A 69-year old Japanese woman with hypertension was admitted because of continuous back pain and recurrent hemoptysis. Radiographic findings showed an enhanced irregular mass, at the aortic arch fed by the tracheal artery, which implied both a penetrating aortic atherosclerotic ulcer and lung cancer. Diagnostic surgery revealed no evidence of cancer but did reveal a rupture of the intima at the distal part of the aortic arch. It is assumed that the transmural oozing occurred after development of the penetrating aortic ulcer, which formed an extra-aortic hematoma and caused surrounding inflammation, and led to tracheal artery feeding. The intramural hematoma might have weakened vascular wall tension from the aorta, and formed an oozing extra-aortic hematoma instead of an acute rupture. (Jpn Heart J 2000; 41: 781-785)
\end{abstract}

Key words: Penetrating aortic ulcer, Extra-aortic hematoma, Hemoptysis, Magnetic resonance imaging

NEW imaging modalities have made it possible to establish the diagnosis of penetrating aortic ulceration with a high degree of accuracy and to tailor management according to the presence of complications. ${ }^{1)}$ Patients with a penetrating aortic ulcer usually present with back or chest pain that mimics classic aortic dissection. The pathologic findings, however, are distinctly different from those of aortic dissection; an atherosclerotic lesion with ulceration penetrates into the internal elastic lamina, allowing hematoma formation within the media of the aortic wall. This may lead to a false aneurysm or, rarely, transmural rupture of the aorta. ${ }^{1)}$

There have been no reports in which a penetrating aortic ulcer has a complication such as an extra-aortic hematoma. In this paper, we report a case diagnosed to have either a penetrating aortic ulcer or lung cancer and was assumed to have a penetrating aortic ulcer with an extra-aortic hematoma.

From the First Department of Medicine, Nippon Medical School, Tokyo, Japan.

Address for correspondence: Yoshihiko Seino, MD, First Department of Medicine, Nippon Medical School, 1-1-5 Sendagi Bunkyo-ku, Tokyo 113-8603, Japan.

Received for publication August 10, 2000.

Revised and accepted August 17, 2000. 


\section{Case}

A 69-year old Japanese woman with hypertension that had been treated with calcium antagonists was admitted to our department because of a one-week history of continuous back pain and recurrent episodes of cough with hemoptysis. Her vital signs upon admission were as follows; temperature, 36.7C; pulse, 86 beats / min, regular; BP, rt 128/844 $\mathrm{mmHg}$, It $120 / 804 \mathrm{mmHg}$; and respiratory rate, $22 / \mathrm{min}$. Physical examination revealed a systolic ejection murmur with Levine 2/6 and maximum strength point at 2RSB, and no vascular bruit. The laboratory tests revealed elevated blood glucose and $\mathrm{HbA} 1 \mathrm{c}$ levels, elevated C-reactive protein concentration, and mild microcytic hypochromic anemia.

Chest x-rays showed an upper shift of the aortic knob. Computed tomography demonstrated an enhanced irregular mass at the distal portion of the aortic arch. Magnetic resonance images of the chest showed a $5.2 \times 2.8 \times 2.8 \mathrm{~cm}$ mass with vascularity on the distal arch (Figure 1) From these findings, the mass lesion was considered to be either an aortic

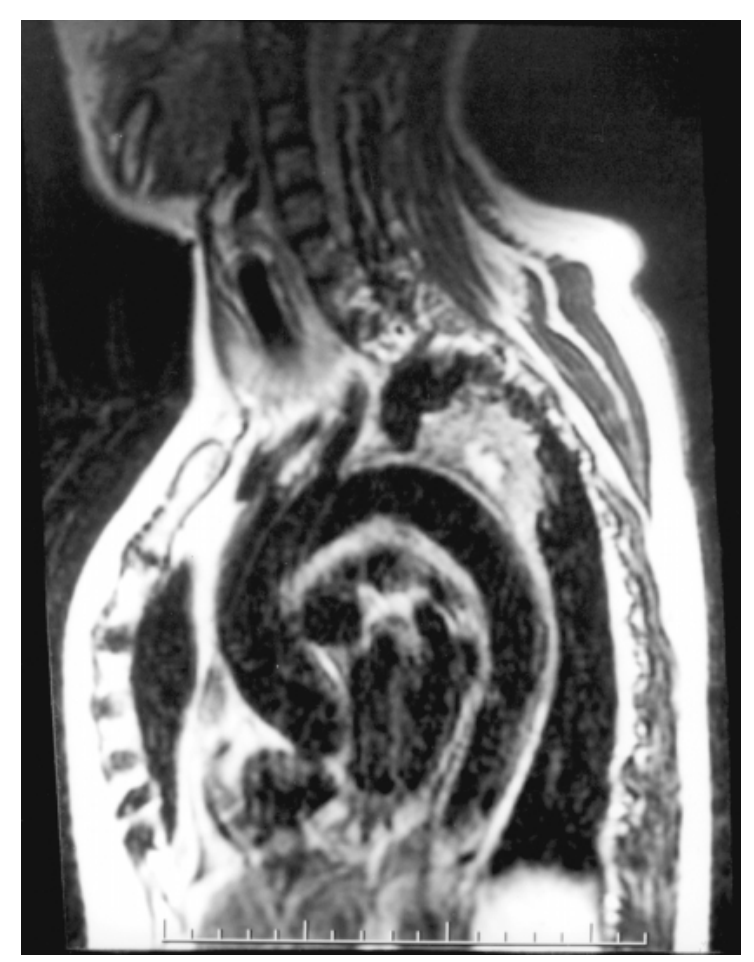

Figure 1. Magnetic resonance image showing an enhanced irregular mass at the distal aortic arch. 
dissection or lung cancer. However, the cytology of the sputum was class 2 with no malignant findings.

The patient was transferred to the ICU to control her blood pressure in case of aortic dissection. The pain and hemoptysis were relieved after the blood pressure was controlled. Further examinations of the lung mass were performed 2 weeks after admission when her blood pressure had become stable.

Magnetic resonance angiography demonstrated a penetrating aortic ulcer with extra-aortic oozing around the distal part of the aortic arch (Figure 2). Tracheal arteriography demonstrated an enhanced irregular mass along with the aortic arch (Figure 3). Since the irregular lung mass fed by the tracheal artery still implied the possibility of lung cancer, exploratory surgery was performed. The mass where the cancer was suspected had shrunk and there was no evidence of cancer. Instead, rupture of the intima was found at a site $3 \mathrm{~cm}$ distal to the aortic arch. Therefore, we

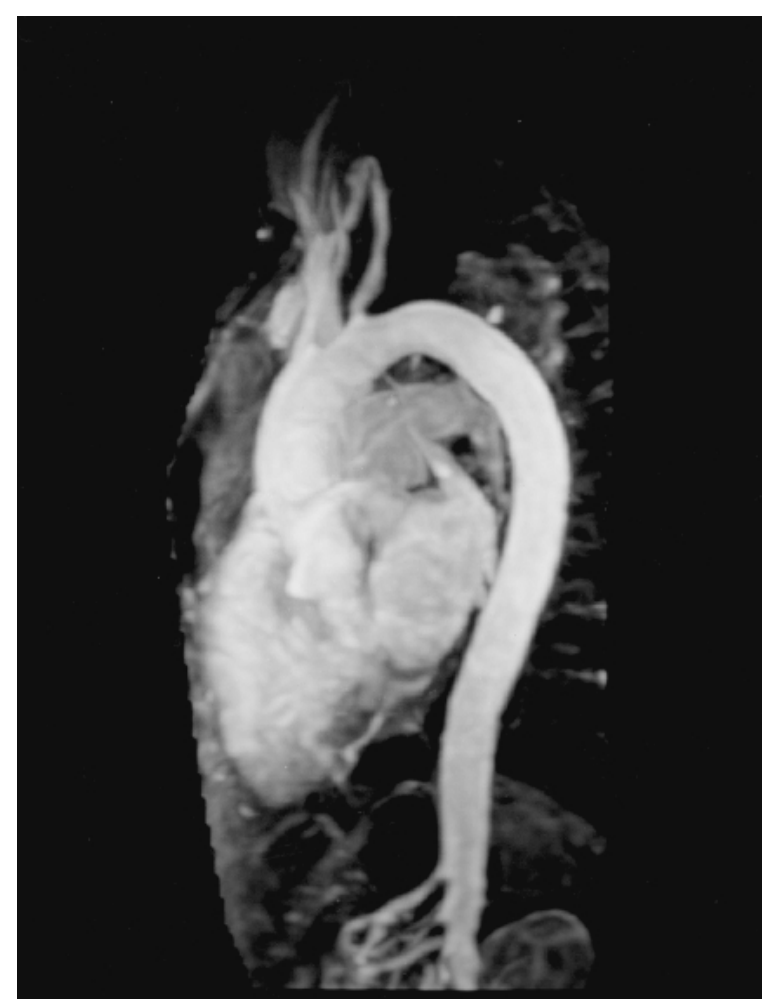

Figure 2. Magnetic resonance angiography demonstrating a penetrating aortic ulcer with extraaortic oozing around the distal part of the aortic arch. 


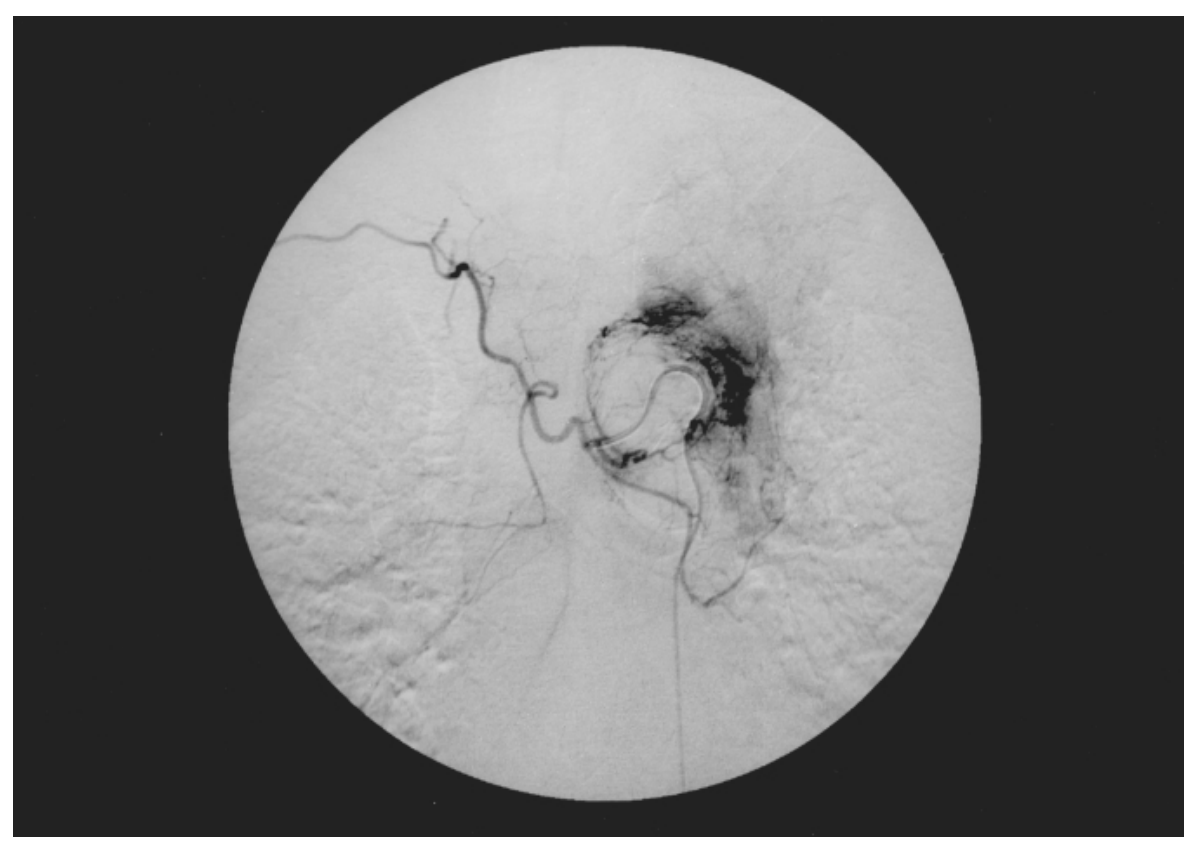

Figure 3. Trachial arteriography showing an enhanced irregular mass fed by the trachial artery along with the aortic arch.

made the diagnosis of a penetrating atherosclerotic ulcer of the aortic arch with an extra-aortic hematoma that was absorbed spontaneously following the stabilization of blood pressure.

\section{DISCUSSION}

A penetrating atherosclerotic ulcer, first defined in the literature in 1986 by Stanson, et $a l,{ }^{2)}$ is an ulceration of an atherosclerotic lesion of the aorta that penetrates into the internal elastic lamina and allows hematoma formation within the media of the aortic wall. The typical patient is elderly with multiple cardiac risk factors and presents with acute chest or back pain. The diagnosis of a penetrating aortic ulcer can be confirmed by computed tomography, magnetic resonance imaging, aortography or transesophageal echocardiography. ${ }^{3)}$ A penetrating aortic ulcer usually occurs in the mid- and distal descending aorta and may be complicated by a transmural aortic rupture, embolization, pseudoaneurysm formation, or progressive aneurysmal dilatation. ${ }^{4)}$

In the present case, an atherosclerotic lesion with ulceration would have penetrated into the internal elastic lamina, allowing initally the for- 
mation of an intramural hematoma and small aneurysm within the media of the aortic wall. However, the formation of an aneurysm alone can not explain the extra aortic mass lesion that was fed by the tracheal artery.

Penetrating aortic ulcers rarely lead to transmural rupture of the aorta, ${ }^{5-6)}$ and no papers showing a penetrating aortic ulcer complicated with an extra-aortic hematoma were found. However, Coady, et al. ${ }^{7)}$ found that intramural hematomas had higher rupture rates than classic aortic dissections, probably because the level of blood collection is more superficial and closer to the adventitia than that of aortic dissection. Since penetrating atherosclerotic ulcers also occur closer to the adventitia, it is not difficult to imagine that they could rupture in some instances.

From these findings, it is assumed, in our case, that the transmural oozing occurred after formation of the intramural hematoma and small aneurysm, which formed an extra aortic hematoma and caused the surrounding inflammation and led to the tracheal artery feeding. The initial symptom of recurrent hemoptysis that disappeared after stabilization of the blood pressure also supports the condition that an oozing hematoma was located in the extra-aorta. The existence of an intramural hematoma might have weakened the vascular wall tension from the aorta, and allowed the formation of an extra aortic hematoma instead of an acute rupture. Since the mass lesion was found to have shrunk during the exploratory surgery, it is more natural to consider that the transmural rupture occurred in terms of oozing. The oozing hematoma would have shrunk following blood pressure stabilization. To our knowledge, this is the first report describing a penetrating aortic ulcer that formed an extra aortic hematoma fed by the tracheal artery.

\section{REFERENCES}

1. Movsowitz HD, Lampert C, Jacobs LE, et al. Penetrating atherosclerotic aortic ulcers. Am Heart J 1994; 128 : 1210-7.

2. Stanson, AW, Kazmier, FJ, Hollier, LH, et al. Penetrating atherosclerotic ulcers of the thoracic aorta: natural history and clinicopathological correlations. Ann Vasc Surg 1986; 1: 15.

3. Vilacosta I, San Roman JA, Aragoncillo P, et al. Penetrating atherosclerotic aortic ulcer: documentation by transesophageal echocardiography. J Am Coll Cardiol 1998; 32: 83-9.

4. Braverman AC. Penetrating atherosclerotic ulcers of the aorta. Current Opinion in Cardiology 1994; 9: 591-7.

5. Welch TJ. Stanson AW. Sheedy PF, et al. Radiologic evaluation of penetrating aortic atherosclerotic ulcer. Radiographics 1990; 10: 675-85.

6. Harris JA, Bis KG, Glover JL, et al. Penetrating atherosclerotic ulcers of the aorta. J Vasc Surg 1994; 19: 90-8.

7. Coady MA, Rizzo JA, Elefteriades JA. Pathologic variants of thoracic aortic dissections. Penetrating atherosclerotic ulcers and intramural hematomas. Cardiology Clinics of North America 1999; 17: 637-57. 